Theoret. Appl. Mech., Vol.34, No.2, pp. 161-171, Belgrade 2007

\title{
Transport properties of a class of electromagnetic waves
}

\author{
David C. Christie *
}

\begin{abstract}
We demonstrate that in many cases, known frame-dependent transport equations for a propagating electromagnetic 2-form in an arbitrarily curved spacetime can be replaced by a much simpler set of frame-independent equations. The frame-dependent equations can then be more easily recovered from the simpler set presented here if required. The relationship of such transport equations to the WKB approximation is also briefly discussed.
\end{abstract}

Keywords: electromagnetic waves, Dehnen, ray transport, gravitational Faraday-effect, differential forms, WKB approximation

\section{Introduction}

Ever since the early tests of general relativity, modelling the behaviour of electromagnetic waves in arbitrary spacetimes has yielded important information. For example, the effect of massive astronomical bodies on the phase and propagation direction of light has given rise to the study of gravitational lensing, which is providing a valuable source of astronomical data. One can also gain useful insights into a gravitational field from its effects on the amplitude and polarisation of an electromagnetic wave. Dehnen [2] managed to derive a set of exact transport equations for the

*Department of Physics, Lancaster University and the Cockcroft Institute, Lancaster, LA1 4YB,UK, e-mail: d.christie@lancaster.ac.uk 
amplitude, polarisation and propagation direction of a plane electromagnetic wave, and was able to use these equations to predict a change in the polarisation of light passing along the axis of a rotating massive ring, known as the "Gravitational Faraday-Effect".

He constructed an electromagnetic 2-form $F$ from a pair of orthogonal 1 -forms, a spacelike $b$ and null $k$. In co-ordinate free notation, this is written

$$
F=b \wedge k
$$

A unit timelike observer vector field $U$ was then introduced. In terms of the electric 1-form $E=\mathrm{i}_{U} F$, the electromagnetic 2-form can then be written

$$
F=-\frac{E \wedge k}{\left(\mathrm{i}_{U} k\right)}
$$

Following the notation in [1], $\mathrm{i}_{X}$ signifies an interior derivative with respect to any vector field $X$. The electric field is decomposed into a unit polarisation 1-form $a$ (which is therefore orthogonal to $\tilde{U}$ and $k$ ) and amplitude $\varepsilon$, viz.

$$
E=\varepsilon a
$$

Taking various contractions of the Maxwell equations and reparameterising $k$ eventually leads to a set of exact transport equations. Reworking his proof in the language of differental forms, it can be shown that his transport equations can be rewritten:

$$
\begin{aligned}
\nabla_{\tilde{k}} k & =0 \\
2 \mathrm{i}_{\tilde{k}} \mathrm{~d} \varepsilon+\varepsilon\left(-2\left(\mathrm{i}_{U} k\right)^{-1} \mathrm{i}_{\tilde{k}} \nabla_{\tilde{k}} \tilde{U}-\delta k\right) & =0 \\
2 h\left(\nabla_{\tilde{k}} a\right)-h\left(\left(\mathrm{i}_{\tilde{k}} \nabla_{X_{a}} a\right) e^{a}+\nabla_{\tilde{a}} k\right) & =0 \\
h((2 \nabla k+\delta k h(g)) \tilde{h}) & =0
\end{aligned}
$$

Here, $\nabla_{X}$ is the covariant derivative with respect to the vector field $X, \delta$ is the operator given by

$$
\delta \equiv \star \mathrm{d} \star
$$

where $\star$ is the Hodge map with respect to the spacetime metric $g$, and $h$ is a $(\underset{\tilde{h}}{1}, 1)$ projection tensor onto a screen plane orthogonal to the wave vector $\tilde{k}$ and observer field $U$ given by

$$
h \equiv \mathbb{I}-\left(\mathrm{i}_{U} k\right)^{-2} k \otimes \tilde{k}-\left(\mathrm{i}_{U} k\right)^{-1} k \otimes U-\left(\mathrm{i}_{U} k\right)^{-1} \tilde{U} \otimes \tilde{k}
$$


where $\mathbb{I}$ is the $(1,1)$ identity tensor in spacetime.

When working in component notation, and adopting a preferred observer field, deriving this set of separated transport equations required a considerable feat of manipulation. However, bringing the powerful modern tool of exterior differental geometry to bear on the problem reveals considerable potential for simplification of both the analysis and the final equations. If we assume that we can describe the wave in such a way that the 1 -form $k$ is closed, so that $\mathrm{d} k=0$, and avoid selecting a preferred observer frame, the Maxwell equations quickly yield a simpler set of transport equations, valid in any frame of reference and possibly more amenable to integration. These will be derived in the next section and are expressed in terms of new frame-independent quantities. We will then go on to show that subsituting Dehnen's quantities into these relations will result in the recovery of his original equations.

\section{Transport Equations}

We write the electromagnetic 2-form in a slightly different notation to Dehnen's as

$$
F=\epsilon k \wedge \mathcal{P}
$$

$\epsilon$ is a scalar amplitude, $k$ is a null wave propagation 1 -form and $\mathcal{P}$ is a real spacelike unit polarisation 1 -form orthogonal to $k$, so that

$$
\mathrm{i}_{\widetilde{k}} k=0 \quad \mathrm{i}_{\widetilde{\mathcal{P}}} \mathcal{P}=1 \quad \mathrm{i}_{\widetilde{k}} \mathcal{P}=0
$$

In contrast to Dehnen's approach, we do not assume that the polarisation 1 -form is orthogonal to the observer field $U$. We make the additional simplifying assumption that $k$ is closed. Thus,

$$
\mathrm{d} k=0
$$

\subsection{Transport Equation for $\mathrm{k}$}

Let $a$ and $b$ represent a pair of 1 -forms with constant scalar product. For arbitrary vector field $Y$,

$$
0=\nabla_{Y}\left(\mathrm{i}_{\widetilde{a}} b\right)=\mathrm{i}_{\widetilde{a}} \nabla_{Y} b+\mathrm{i}_{\tilde{b}} \nabla_{Y} a
$$


Thus, a 1-form $\varphi$ with constant norm has the property

$$
\mathrm{i}_{\widetilde{\varphi}} \nabla_{Y} \varphi=0 \quad \forall Y \in \Gamma T M
$$

In torsion-free spacetime with arbitrary dual bases $\left\{e^{a}\right\}$ and $\left\{X_{a}\right\}$, the exterior derivative operator can be expressed [1]

$$
d=e^{a} \wedge \nabla_{X_{a}}
$$

For arbitrary vector fields $Y$ and $Z$ and arbitrary 1-form $\omega$, (15) leads to the relation

$$
\mathrm{i}_{Y} \mathrm{i}_{Z} \mathrm{~d} \omega=\mathrm{i}_{Y} \nabla_{Z} \omega-\mathrm{i}_{Z} \nabla_{Y} \omega
$$

Let $\gamma$ represent an arbitrary closed 1-form of constant norm, and $Y$ represent an arbitrary vector field. As $\gamma$ is closed,

$$
\begin{aligned}
0 & =\mathrm{i}_{Y} \mathrm{i}_{\widetilde{\gamma}} \mathrm{d} \gamma=\mathrm{i}_{Y} \nabla_{\widetilde{\gamma} \gamma}-\mathrm{i}_{\widetilde{\gamma}} \nabla_{Y} \gamma \\
& =\mathrm{i}_{Y} \nabla_{\widetilde{\gamma}} \gamma
\end{aligned}
$$

due to (14). Since $Y$ is an arbitrary vector field, we have

$$
\nabla_{\widetilde{\gamma}} \gamma=0
$$

Thus, any closed 1-form of constant norm is parallel transported along the integral curves of its metric dual. Hence, the closed, null 1-form $k$ will obey the transport equation

$$
\nabla_{\widetilde{k}} k=0
$$

\subsection{Transport Equation for $\epsilon$}

Equation (15) can be used to show that, for arbitrary 1-forms $\alpha$ and $\beta$ and arbitrary 0 -form $f$,

$$
\delta(f \alpha \wedge \beta)=f\left\{(\delta \alpha) \beta-(\delta \beta) \alpha+\nabla_{\widetilde{\beta}} \alpha-\nabla_{\widetilde{\alpha}} \beta\right\}+\left(\mathrm{i}_{\widetilde{\mathrm{d} f}} \alpha\right) \beta-\left(\mathrm{i}_{\widetilde{\mathrm{d} f}} \beta\right) \alpha
$$

Thus, the vacuum Maxwell equations can be written

$$
\begin{aligned}
& 0=\mathrm{d} F=\mathrm{d} \epsilon \wedge k \wedge \mathcal{P}-\epsilon k \wedge \mathrm{d} \mathcal{P} \\
& \left.0=\delta F=\epsilon\left\{(\delta k) \mathcal{P}-(\delta \mathcal{P}) k+\nabla_{\widetilde{\mathcal{P}}} k-\nabla_{\widetilde{k}} P\right\}+\left(\mathrm{i}_{\widetilde{\mathcal{P}}} \mathrm{d} \epsilon\right) k-\left(\mathrm{i}_{\widetilde{k}} \mathrm{~d} \epsilon\right) \mathcal{P} 22\right)
\end{aligned}
$$


Contracting (21) with $\widetilde{k}$ and then $\widetilde{\mathcal{P}}$ gives

$$
0=\left(\mathrm{i}_{\widetilde{k}} \mathrm{~d} \epsilon\right) k+\left(\epsilon \mathrm{i}_{\widetilde{\mathcal{P}}} \mathrm{i}_{\widetilde{k}} \mathrm{~d} \mathcal{P}\right) k
$$

Recalling (16), one can write

$$
\begin{aligned}
\mathrm{i}_{\widetilde{k}} \mathrm{~d} \epsilon & =-\epsilon\left(\mathrm{i}_{\widetilde{\mathcal{P}}} \nabla_{\widetilde{k}} \mathcal{P}-\mathrm{i}_{\widetilde{k}} \nabla_{\widetilde{\mathcal{P}}} \mathcal{P}\right) \\
& =-\epsilon \mathrm{i}_{\widetilde{\mathcal{P}}} \nabla_{\widetilde{\mathcal{P}}} k
\end{aligned}
$$

where the last line is a consequence of (13) and (14). Now, contracting (22) with $\widetilde{\mathcal{P}}$ and applying (14) gives

$$
0=\epsilon\left\{(\delta k)+\mathrm{i}_{\widetilde{\mathcal{P}}} \nabla_{\widetilde{\mathcal{P}}} k\right\}-\left(\mathrm{i}_{\widetilde{k}} \mathrm{~d} \epsilon\right)
$$

Combining (24) and (25) gives the transport equation for the amplitude of the wave:

$$
\mathrm{i}_{\widetilde{k}} \mathrm{~d} \epsilon=\frac{1}{2} \epsilon \delta k
$$

\subsection{Transport Equation for $\mathcal{P}$}

Taking the wedge product of $(21)$ with $\mathcal{P}$ and then contracting with $\widetilde{k}$ gives

$$
0=k \wedge \mathrm{i}_{k} \mathrm{~d} \mathcal{P} \wedge \mathcal{P}
$$

Furthermore, as $k$ is closed, (13) and (14) give the following relations, where $X$ is an arbitrary vector field.

$$
\begin{aligned}
\mathrm{i}_{X} \mathrm{i}_{\widetilde{k}} \mathrm{~d} \mathcal{P} & =\mathrm{i}_{X} \nabla_{\widetilde{k}} \mathcal{P}-\mathrm{i}_{\widetilde{k}} \nabla_{X} \mathcal{P} \\
0=\mathrm{i}_{X} \mathrm{i}_{\widetilde{\mathcal{P}}} \mathrm{d} k & =\mathrm{i}_{X} \nabla_{\widetilde{\mathcal{P}}} k-\mathrm{i}_{\widetilde{\mathcal{P}}} \nabla_{X} k \\
& =\mathrm{i}_{X} \nabla_{\widetilde{\mathcal{P}}} k+\mathrm{i}_{\widetilde{k}} \nabla_{X} \widetilde{\mathcal{P}}
\end{aligned}
$$

Adding together (28) and (29) gives

$$
\begin{aligned}
\mathrm{i}_{X} \mathrm{i}_{\widetilde{k}} \mathrm{~d} \mathcal{P} & =\mathrm{i}_{X} \nabla_{\widetilde{k}} \mathcal{P}+\mathrm{i}_{X} \nabla_{\widetilde{\mathcal{P}}} k \quad \forall X \in \Gamma T M \\
\Rightarrow \mathrm{i}_{\widetilde{k}} \mathrm{~d} \mathcal{P} & =\nabla_{\widetilde{k}} \mathcal{P}+\nabla_{\widetilde{\mathcal{P}}} k
\end{aligned}
$$

Now, taking the wedge product of (22) with $k$ and eliminating $\mathrm{i}_{\widetilde{k}} \mathrm{~d} \epsilon$ using

(25) gives

$$
0=-\left(\mathrm{i}_{\widetilde{\mathcal{P}}} \nabla_{\widetilde{\mathcal{P}}} k\right) \mathcal{P} \wedge k+\nabla_{\widetilde{\mathcal{P}}} k \wedge k-\nabla_{\widetilde{k}} \mathcal{P} \wedge k
$$


or

$$
0=\mathrm{i}_{\widetilde{\mathcal{P}}}\left\{\mathcal{P} \wedge \nabla_{\widetilde{\mathcal{P}}} k \wedge k\right\}-\nabla_{\widetilde{k}} \mathcal{P} \wedge k
$$

Applying (30), (32) can be rewritten

$$
0=\mathrm{i}_{\widetilde{\mathcal{P}}}\left\{P \wedge \mathrm{i}_{\widetilde{k}} \mathrm{~d} \mathcal{P} \wedge k\right\}-2 \nabla_{\widetilde{k}} \mathcal{P} \wedge k
$$

The first term in this equation vanishes due to (27), leaving

$$
0=\nabla_{\widetilde{k}} \mathcal{P} \wedge k
$$

which is equivalent to

$$
\nabla_{\widetilde{k}} \mathcal{P}=\beta k
$$

for some arbitrary 0 -form $\beta$. This can be further simplified with a reparameterisation. ${ }^{1}$ Replacing $\mathcal{P}$ with $\mathcal{P}^{\prime}=\mathcal{P}+\sigma k$ for arbitrary 0 -form $\sigma$ will not affect (10), (21) or (22). (35) becomes

$$
\nabla_{\widetilde{k}} \mathcal{P}^{\prime}=\nabla_{\widetilde{k}} \mathcal{P}+\widetilde{k}(\sigma) k=(\beta+\widetilde{k}(\sigma)) k=0 \quad \text { if } \tilde{k}(\sigma)=-\beta
$$

While these transport equations have been derived for a real polarisation 1 -form, it is straightforward to obtain the same equations when $P$ is a complex 1-form that satisfies $g(\mathcal{P}, \overline{\mathcal{P}})=1$.

\subsection{Summary of Results}

A family of solutions to Maxwell's equations can be written

$$
F=\epsilon k \wedge \mathcal{P}
$$

where

$$
\mathrm{d} k=0 ; \quad \mathrm{i}_{\widetilde{k}} k=\mathrm{i}_{\widehat{k}} \mathcal{P}=0 ; \quad \mathrm{i}_{\widetilde{\mathcal{P}}} \mathcal{P}=1
$$

Given initial conditions on $F$, its evolution can be calculated by integrating the following simple transport equations:

$$
\begin{array}{r}
\nabla_{\widetilde{k}} k=0 \\
\mathrm{i}_{\widetilde{k}} \mathrm{~d} \epsilon=\frac{1}{2} \epsilon \delta k \\
\nabla_{\widetilde{k}} \mathcal{P}=0
\end{array}
$$

\footnotetext{
${ }^{1}$ Such reparameterisations are common in these calculations. Dehnen's formulation allows him to reparameterise his wave vector $k$ to obtain its transport equation. Similarly, in [5], a reparameterisation of the 'complex amplitude' 1-form simplifies its WKB transport equation, allowing decomposition into polarisation and amplitude parts.
} 


\section{Recovering Dehnen's Equations}

Define a timelike unit vector field $U$ whose integral curves correspond to observer worldlines $(g(U, U)=-1)$. For our electromagnetic 2-form in (36), we can define an electric field by

$$
E=\mathrm{i}_{U} F=\epsilon\left\{\left(\mathrm{i}_{U} k\right) \mathcal{P}-\left(\mathrm{i}_{U} \mathcal{P}\right) k\right\}
$$

Comparing (2) and (3) with (36) and (41) gives the relations

$$
\varepsilon=\left(\mathrm{i}_{U} k\right) \epsilon ; \quad a=\mathcal{P}-\frac{\left(\mathrm{i}_{U} \mathcal{P}\right)}{\left(\mathrm{i}_{U} k\right)} k
$$

Dehnen's polarisation $a$ is therefore a projection of $\mathcal{P}$ onto the hyperplane orthogonal to the observer field $U$, ensuring that $\mathrm{i}_{U} a=0$. We can use these relations to recover Dehnen's original equations from (38)-(40). Firstly, note that $k$ is the same as Dehnen's so that (4) follows immediately from (40). Now, making use of (39) and (42) and noting that $\nabla_{\widetilde{k}} k=0$ gives

$$
\begin{aligned}
2 \mathrm{i}_{\widetilde{k}} \mathrm{~d} \varepsilon & =2 \mathrm{i}_{\widetilde{k}} \mathrm{~d}\left(\left(\mathrm{i}_{U} k\right) \epsilon\right) \\
& =2 \mathrm{i}_{\tilde{k}} \mathrm{~d} \epsilon\left(\mathrm{i}_{U} k\right)+2 \epsilon\left(\mathrm{i}_{\tilde{k}} \mathrm{~d}\left(\mathrm{i}_{U} k\right)\right) \\
& =\left(\mathrm{i}_{U} k\right) \epsilon \delta+2 \epsilon \widetilde{k}\left(\mathrm{i}_{U} k\right) \\
& =\left(\mathrm{i}_{U} k\right) \epsilon \delta k+2 \epsilon\left(\mathrm{i}_{U} \nabla_{\widetilde{k}} k+\mathrm{i}_{\widetilde{k}} \nabla_{\widetilde{k}} \tilde{U}\right) \\
& =\varepsilon\left(\delta k+2\left(\mathrm{i}_{U} k\right)^{-1} \mathrm{i}_{\tilde{k}} \nabla_{\widetilde{k}} \tilde{U}\right)
\end{aligned}
$$

so that we recover (5). Now, writing

$$
\zeta \equiv \frac{\left(\mathrm{i}_{U} \mathcal{P}\right)}{\left(\mathrm{i}_{u} k\right)}
$$

so that

$$
a=\mathcal{P}-\zeta k
$$

we have

$$
\begin{aligned}
h\left(\nabla_{\widetilde{k}} a\right) & =h\left(\nabla_{\widetilde{k}}(\mathcal{P}+\zeta k)\right. \\
& =h\left(\nabla_{\widetilde{k}} \mathcal{P}\right)+\zeta h\left(\nabla_{\widetilde{k}} k\right)+\widetilde{k}(\zeta) h(k) \\
& =0
\end{aligned}
$$


as $\nabla_{\widetilde{k}} \mathcal{P}=0$ from (40), $\nabla_{\widetilde{k}} k=0$ from (38) and $h(k)=0$ from the properties of $h$. This differs from (6) due to the simplifying assumption that $k$ is closed. We can write

$$
0=\mathrm{i}_{\widetilde{a}} \mathrm{~d} k=\left(\mathrm{i}_{\widetilde{a}}\left(e^{a} \wedge \nabla_{X_{a}} k\right)=\nabla_{\widetilde{a}} k-\mathrm{i}_{\widetilde{a}} \nabla_{X_{a}} k=\nabla_{\widetilde{a}} k+\mathrm{i}_{\tilde{k}} \nabla_{X_{a}} a\right.
$$

Thus,

$$
h\left(\nabla_{\widetilde{a}} k+\mathrm{i}_{\tilde{k}} \nabla_{X_{a}} a\right)=0
$$

We can therefore recover (6).

Finally, note that ((14) and (38) imply that

$$
\mathrm{i}_{\widetilde{a}} \nabla_{\widetilde{a}} k=\mathrm{i}_{\widetilde{\mathcal{P}-\zeta k}} \nabla_{\widetilde{\mathcal{P}-\zeta k}} k=\mathrm{i}_{\widetilde{\mathcal{P}-\zeta k}} \nabla_{\widetilde{\mathcal{P}}} k=\mathrm{i}_{\widetilde{\mathcal{P}}} \nabla_{\widetilde{\mathcal{P}}} k
$$

so that (24) and (25) give

$$
0=\delta k+2 \mathrm{i}_{\widetilde{\mathcal{P}}} \nabla_{\widetilde{\mathcal{P}}} k=\delta k+2 \mathrm{i}_{\widetilde{a}} \nabla_{\widetilde{a}} k
$$

As $h(g)$ is the projection into the screen plane orthogonal to $U$ and $k$ of the metric tensor, we have

$$
h(g)(\widetilde{a}, \widetilde{a})=1
$$

so that (50) can be rewritten

$$
\{\delta k h(g)+\nabla k\}(\widetilde{a}, \widetilde{a})=0
$$

As the term in the brace brackets is not antisymmetric, and $\widetilde{a}$ is an arbitrary spacelike "screen plane" vector field, this implies that

$$
h(\{\delta k h(g)+\nabla k\} \tilde{h})=0
$$

and we have recovered the final Dehnen transport equation (7).

\section{Discussion}

We have therefore demonstrated that if we avoid adopting a preferred reference frame from the outset, a simpler set of transport equations can be derived for the polarisation, amplitude and wave vector of a plane 
electromagnetic wave if the wave vector satisfies $\mathrm{d} k=0$. This condition is often true in physical situations. For example, in the second half of Dehnen's paper, he applies his transport equations to an electromagnetic wave in the axis of a rotating massive cylinder. The wave 1-form he derives is closed. In fact, it is exact (i.e. $k=\mathrm{d} S$ for some function $S$ ) and can be obtained by solving, with initial conditions, the partial differential equation $\mathrm{i}_{\tilde{d} S} \mathrm{~d} S=0$, which then automatically ensures the transport equation is satisfied. This can be more straightforward than integrating the transport equation directly.

The new set of equations derived in Part 2 are easier to integrate from initial conditions than the original transport equations, and the resulting values for $\mathcal{P}, k$ and $\epsilon$ are independent of any frame of reference. The observer field $U$ can then finally be introduced in the usual way by constructing the electromagnetic 2-form from (36) and then taking

$$
E=\mathrm{i}_{U} F ; \quad B=-\mathrm{i}_{U} * F
$$

Finally, it is noteworthy that an electromagnetic 2-form similar to (36) also emerges from a quite different treatment of the behaviour of electromagnetic waves in arbitrary metrics, the short-wavelength WKB approximation. This is described in $[4,5]$, while [3] considers propagation in a medium. A complex approximate solution to the Maxwell equations can be written

$$
F=\Re\left\{e^{\frac{i}{\xi} S}\left(A+\frac{\xi}{i} B+\mathcal{O}\left(\xi^{2}\right)\right)\right\}
$$

where $\xi$ is a small parameter. This represents an electromagnetic wave that is locally plane and monochromatic over a distance scale significantly greater than its wavelength, but far smaller than the radius of curvature of the spacetime. To first order in $\xi$, one can write

$$
F=\Re\left\{\alpha e^{i S} \mathrm{~d} S \wedge \mathcal{P}\right\}
$$

where $\mathrm{d} S$ is a real null 1 -form that is orthogonal to the complex unit polarisation form $\mathcal{P}$ and $\alpha$ is a real amplitude scalar. These quantities obey the WKB transport equations

$$
\nabla_{\tilde{\mathrm{d} S}} \mathrm{~d} S=0 \quad \mathrm{i}_{\mathrm{d} S} \mathrm{~d} \alpha=\frac{1}{2}(\delta \mathrm{d} S) \alpha \quad \nabla_{\tilde{\mathrm{d} S}} \mathcal{P}=0
$$


These WKB equations were obtained using an approximation method, and their validity depends on the wavelength of the light and the properties of the surrounding space-time. However, we can construct the electromagnetic 2-form (56) by substituting $k=\mathrm{d} S$ and $\epsilon=\alpha e^{i \mathrm{~d} S}$ in (36) and allowing $\mathcal{P}$ to be complex. Our transport equations (38)-(40), which did not require any approximation scheme, are then identical to the WKB equations. Thus, if the wave is exactly described by (56), the WKB transport equations are exact, rather than approximate, evolution equations.

\section{Acknowledgements}

This research was supported by the Cockcroft Institute and Lancater University. The author is grateful to Professor R.W. Tucker for useful discussions.

\section{References}

[1] I. M. Benn and R. W. Tucker, An Introduction to Spinors and Geometry with Applications in Physics, Hilger, Bristol 1987

[2] H. Dehnen, Gravitational Faraday-Effect, International Journal of Theoretical Physics, Vol 7 No 6 (1973) 467-474

[3] J. Ehlers, Zum Übergang von der Wellenoptik zur geometrischen Optik in der allgemeinen Relativitätstheorie, Zeitschrift für Naturforschung 22A (1967) 1328-1332

[4] C. W. Misner, K. S. Thorne and C. W. Wheeler, Gravitation, W. H. Freeman, San Fransisco, 1973

[5] P. Schneider, J. Ehlers, E. E. Falco, Gravitational Lenses, SpringerVerlag, Berlin 1992

Submitted on July 2007. 


\section{Transportne osobine jedne klase elektromagnetskih talasa}

Pokazano je da se u mnogim slučajevima poznate - zavisne od sistema referencije - transportne jednačine prostiranja elektromagnetnih 2-formi mogu zameniti mnogo prostijim skupom jednačina nezavisnih od sistema referencije. Ako je potrebno, jednačine zaavisne od sistema referencije se tada mogu lakše ponovo dobiti iz ovde prikazanog sistema. Takodje se kratko diskutuje relacija takvih transportnih jednačina sa WKB aproksmacijom. 